\title{
Subcutaneous pre-sternal tunneling of contra lateral left ventricular lead of CRTP
}

\begin{abstract}
The right ventricular (RV) apex has been the standard pacing site since the development of implantable pacemaker technology. Although RV pacing was initially only utilized for the treatment of severe Brady arrhythmias usually due to complete heart block, today the indications for and implantation of RV pacing devices is dramatically larger
\end{abstract}

Volume 8 Issue 3 - 2017

\author{
Ahmed Adel Anwer Shaheen \\ Assistant Consultant Adult Cardiology, Saudi Arabia \\ Correspondence: Ahmed Adel Anwer Shaheen, Assistant \\ Consultant Adult Cardiology, King Faisal Cardiac Center, King \\ Abdulaziz Medical City, Jeddah, Kingdom of Saudi Arabia,Tel \\ 966544501865, Email dr.ahmed_2009@icloud.com
}

Received: January 09, 2017 | Published: February 24, 2017

Abbreviation: RV, right ventricle; HTN, hypertension; IHD, ischemic heart disease; PCI, percutaneous coronary intervention; $\mathrm{LAD}$, left anterior descending artery; EF, ejection fraction; CS, coronary sinus; DDDR, dual chamber pacemaker with rate mode; LV, left ventricle CRTP, cardiac resynchronization therapy

\section{Introduction}

Recently, the adverse effects of chronic RV apical pacing have been described including an increased risk of heart failure and death. In light of the growing number of implanted devices and enhanced survival of affected patients, the rate of surgical re-interventions at the site of initial system implantation will inevitable rise with time. These procedures will include simple generator replacements, e.g. in cases of battery depletion, but they will also consist of more complex procedures that require the implantation of a new lead, e.g. in case of lead failure, or even of multiple leads, e.g. if a device upgrade is planned. Tran venous lead placement from the site of The initial device implantation is favored for practical considerations, but it may become challenging or even impossible if the respective vein is significantly obstructed or occluded, which may be the case in 5-26\% of the patients with a chronically implanted device by. ${ }^{1,2}$ One potential option in case of ipsilateral vein obstruction is transvenous electrode placement from the contralateral vein with subcutaneous pre-Sternal tunneling (TUN) of the lead to the device. This approach has been described first by Belott. ${ }^{3}$ Although only acute experience from single cases provides evidence for this approach, ${ }^{4}$ it is listed as one potential options in current standard textbooks.

\section{Case report}

62 years old lady presented to the electrophysiology clinic with a history of shortness of breath, orthopnea, paroxysmal nocturnal dyspnea and lower limb swelling have started 3weeks back. She denied any chest pain or syncope. She is known as HTN, IHD (S/P PCI to LAD 10years ago), and History of Complete heart block (S/P DDDR pacemaker 6years back). Previous LV function was normal (EF $>55 \%$, No significant valvular disease). On examination: She was conscious, oriented and vitally stable BP: 110/70 HR 70B/M, regular, RR: 22, Pso2: $96 \%$ on room air, T : 36.5 , Congested neck vein observed, Chest: bilateral fine end in inspiratory basal crackles CVS: Normal S1, S2, no add sound or murmur Abdomen: Lax, soft, no masses Lower limb: Bilateral pitting edema ECG: well functioning paced rhythm; patient was diagnosed as new acute decompensate heart failure which was confirmed by ECHO showed: EF $<35 \%$, with an impressive ventricular dyssynchrony, no significant valvular lesion, The patient was admitted in CCU as a case of ADHF (as a complication of RV pacing), was started on Ant failure treatment and the decision was taken to upgrade the DDDR TO CRTP. Then, Patient was driven in to Catha Lab. The procedure was initially attempted on the right, with successful cannulation of the right subclavian vein but unable to advance the guide wire to the superior vena cava (SVC), Venogram through the right anti-cubital vein showed a short occlusion of the right subclavian vein (Figure 1). The procedure was abandoned and LV lead implantation was performed on the left (Figure 2). Mild dissection of the coronary sinus occurred (Figure 3). Then sealed spontaneously and the LV lead was secured deeply in the posterolateral CS branch, a pair of long forceps were used for blunt dissection to create a subcutaneous tract from the right to the left. The proximal end of the LV lead was grasped with the forceps and tunneled above the sternum to rejoin the pocket (Figure 4). Then programming was done with good homeostasis.

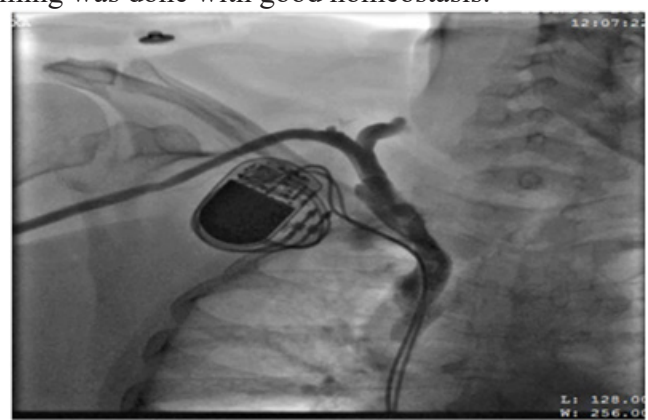

Figure I

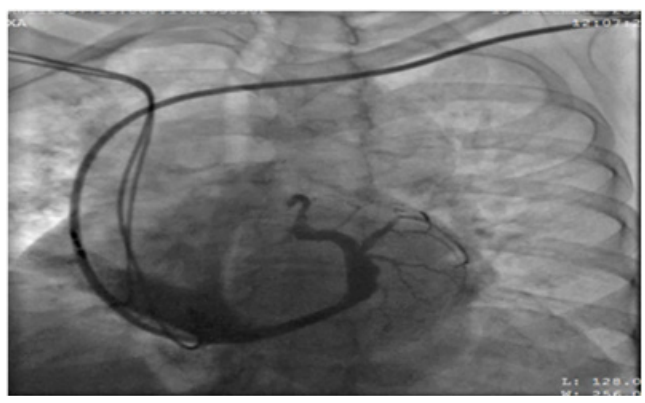

Figure 2 


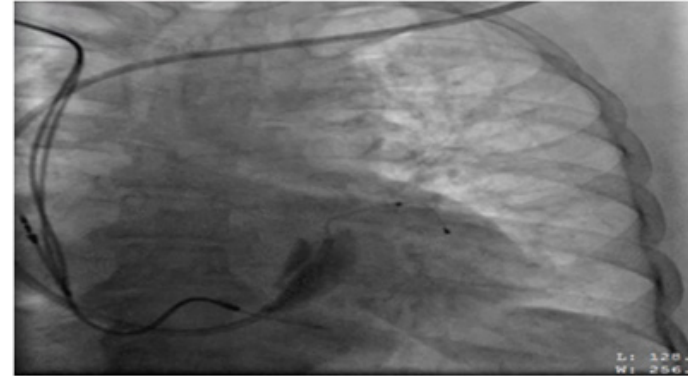

Figure 3

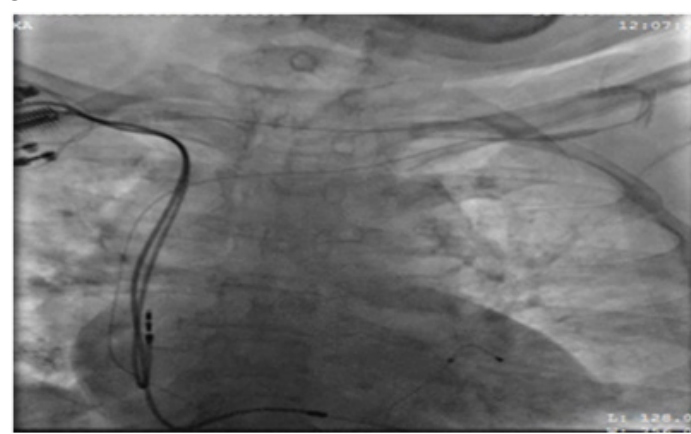

Figure 4

\section{Discussion}

Many reviews detailed the detrimental effects of RV apical pacing and their shared hemodynamic pathophysiology. In particular, the role of RV apical pacing induced ventricular dyssynchrony is highlighted with a specific focus on differential outcome based upon QRS morphology at implant. For difficult cannulation, many alternative routes have been proposed:

1. Puncture of the ipsilateral subclavian or brachiocephalic vein proximal (or medial) to the site of obstruction, with or without a supraclavicular approach with subcutaneous tunneling; ${ }^{6}$

2. Ovadia et al., ${ }^{7}$ describe a Percutaneous brachiocephalic (innominate) or deep subclavian venous access by a supraclavicular approach using an 18-gauge Deseret angiocath, a Terumo Glide wire, and dilation to permit one or two 9-11 Fr sheaths, followed by tunneling of electrodes (pre or retroclavicularly) to a pre or retro pectoral pocket;

3. Alek sic et al., ${ }^{8}$ describe the 'notch technique' to puncture of the contralateral innominate vein (with subcutaneous tunneling) - an $18 \mathrm{~F}$ needle was introduced $1 \mathrm{~cm}$ above the sternoclavicular joint palpable as a notch and angled at $45^{\circ}$ to puncture the innominate vein at $3-4 \mathrm{~cm}$ depth. A $8.5 \mathrm{~F}$ Percutaneous sheath introducer set (Arrow International Inc.) was used and electrode placed with over the wire technique and lead brought back to the original pocket;

4. puncture of the ipsilateral jugular vein from the neck (with subcutaneous tunneling of the lead) is described by Bosa-Odeja et al., ${ }^{9}$ Seldinger technique was used to puncture the jugular vein and a 0.38 inch Teflon guide wire was advanced through the needle to the right ventricle and was replaced with an $8 \mathrm{~F}$ sheath to continue the procedure in the standard fashion. The lead and the protective sheath were secured to the muscle layers in the clavicular portion of sternocleidomastoid muscle

5. Extraction of the defect lead with ipsilateral implantation of a new transvenous electrode by, ${ }^{10}$
6. Trans atrial or epicardial lead placement, ${ }^{11,12}$

7. Contralateral implantation of the complete system with explanation or abandoning of the old leads ${ }^{13}$

8. Fox et al., ${ }^{14}$ describe the procedure using a pair of long forceps and blunt dissection to create a subcutaneous tract from the right to the left and

9. Another technique is the placement of transvenous electrode from the contralateral side with subcutaneous, pre-sternal lead tunneling. Luthje et al described using regular chest tube and a trocar for this technique, and passing the lead in the chest tube to the other end. Thus, contralateral lead placement and subcutaneous pre-sternal TUN (like in our case) can be considered as a feasible option in a broad variety of patients. The optimal surgical approach, however, should be individualized for each subject.

\section{Acknowledgments}

None.

\section{Conflicts of interest}

Author declares there is no conflicts opf interest.

\section{Funding}

None.

\section{References}

1. Haghjoo M, Nikoo M, Fazelifar A, et al. Predictors of venous obstruction following pacemaker or implantable cardioverter-defibrillator implantation: a contrast venographic study on 100 patients admitted for generator change, lead revision, or device upgrade. Europace . 2007;9(5):328-332.

2. Rozmus G, Daubert J, Huang D, et al. Venous thrombosis and stenosis after implantation of pacemakers and defibrillators. J Interv Card Electrophysiol . 2005;13(1):9-19.

3. Belott P. Use of the contralateral subclavian vein for placement of atrial electrodes in chronically VVI paced patients. Pacing Clin Electrophysiol . 1983;6(4):781-783.

4. Fox D, Petkar S, Davidson N, et al. Upgrading patients with chronic defibrillator leads to a biventricular system and reducing patient risk: contralateral LV lead placement. Pacing Clin Electrophysiol. 2006;29(9):1025-1027.

5. Ellenbogen K, Kay G, Lau C, et al. Permanent pacemaker and implantable cardioverter-defibrillator infectio: a population based study. Arch Intern Med. 2007;167(7):669-675.

6. Antonelli D, Freedd berg N, Turgeman Y. Supraclavicular vein approach to overcoming ipsilateral chronic subclavian vein obstruction during pacemaker-ICD lead revision or upgrading. Europace. 2010;12(11):1596-1599.

7. Ovadia M, Cooper RS, Parnell VA, et al. Transvenous pacemaker insertion ispilateral to chronic subclavian vein obstruction: an operative technique for children and adults. Pacing Clin Electrophysio. 2000; 123(11):1585-1593.

8. Aleksic I, Kottenberg-Assenmacher E, Kienbaum P, et al. The innominate vein as alternative venous access for complicated implantable cardioverter defibrillator revisions. Pacing Clin Electrophysiol. 2007;30(8):957-960.

9. Bosa-Odeja F, Bethencourt-Munoz M, Vergara-Torres M, et al. Upgrade of a pacemaker defibrillator to a biventricular device: the internal jugular approach in a case of bilateral subclavian veins occlusion. $J$ Interv Cardiac Electrophysiol. 2007;19(3):209-211. 
10. Bracke F, van Gelder L, Sreeram N, et al. Exchange of pacing or defibrillator leads following laser sheath extraction of non-functional leads in patients with ipsilateral obstructed venous access. Heart. 2000;83(6):12.

11. Jaroszewski D, Altemose G, Scott L, et al. Nontraditional surgical approaches for implantation of pacemaker and cardioverter defibrillator systems in patients with limited venous access. Ann Thorasic Surg . 2009;88(1):112-116.
12. Molina J. Surgical options for endocardial lead placement when upper veins are obstructed or non usable. J Interv Cardiac Electrophysiol. 2004; $11: 149-154$.

13. Luthje L, Zabel M, Seegers D, et al. Acute and long term feasibility of contralateral transvenous lead replacement with sub-coetaneous, pre-Sternal tunnelling in patients with chronically implanted rhythm devices. Europace. 2011;13:100. 